\title{
Postoperative Spine Infections
}

\author{
Abhijit Yuvaraj Pawar ${ }^{1,2}$, Samar Kumar Biswas ${ }^{1}$ \\ ${ }^{I}$ Department of Orthopedics, D.Y. Patil Medical College, Pune, India \\ ${ }^{2}$ Department of Orthopedics, Ruby Hall Clinic, Pune, India
}

Postoperative spinal wound infection increases the morbidity of the patient and the cost of healthcare. Despite the development of prophylactic antibiotics and advances in surgical technique and postoperative care, wound infection continues to compromise patient outcome after spinal surgery. Spinal instrumentation also has an important role in the development of postoperative infections. This review analyses the risk factors that influence the development of postoperative infection. Classification and diagnosis of postoperative spinal infection is also discussed to facilitate the choice of treatment on the basis of infection severity. Preventive measures to avoid surgical site (SS) infection in spine surgery and methods for reduction of all the changeable risk factors are discussed in brief. Management protocols to manage SS infections in spine surgery are also reviewed.

Keywords: Spine surgery; Postoperative infections

\section{Introduction}

Postoperative spinal wound infection is a potentially devastating complication after spine procedures. Despite the development of prophylactic antibiotics and advances in surgical technique and postoperative care, wound infection continues to compromise patients' outcome after spinal surgery [1]. This kind of infection places the patient at risk for pseudoarthrosis, adverse neurologic sequelae, chronic pain, deformity and even death.

A study by Veeravagu et al. [2] shows that patients with a wound infection after an intervention on the spine have a longer hospital stay, higher mortality and higher rates of return to the operation room (OR), as compared to those without surgical wound infections. In addition these infections represent an additional cost to health care; Thalgott et al. [3] report an average additional cost of $\$ 200.000$ for each of these patients.

\section{Incidence}

Surgical site infection (SSI) is the most common hospital acquired infection that occurs in the early postoperative period [4]. Reported rates of infection after spinal interventions vary widely from $0.7 \%$ to $16 \%$ [1-7]; the most important reason for this wide range is that different types of spinal surgery have different risks for postoperative infection due to the differing invasiveness of the procedures. Low invasive interventions, such as discectomy, have the lower incidence of postoperative infections, whereas more invasive techniques, as fusion with instrumentation, have the highest rates of postoperative infections [1,8-10]. Besides the higher invasiveness, the use of instrumentation has an important role in the development of postoperative infections. Furthermore, it can cause local soft tissue irritation leading to inflammation and seroma formation that subsequently provides a fertile breeding ground for microorganisms to grow. Adherence of bacteria to

Received May 12, 2015; Accepted May 13, 2015

Corresponding author: Abhijit Yuvaraj Pawar

Department of Orthopedics, D.Y. Patil Medical College,

Sant Tukaram Nagar Pimpri, Pune, Maharashtra 411001, India

Tel: +91-73-8708-0391, Fax: +91-020-2780-5217, E-mail: drabhijitpawar@gmail.com 
the surface of implants is promoted by a polysaccharide biofilm called glycocalyx that acts as barrier against host defense mechanisms and antibiotics [8,11]. Finally, metallosis from micromotion of the instrumentation leads to granuloma formation and provides yet another medium for bacterial colonization $[8,12]$.

The most recent National Nosocomial Infections Surveillance Summary System, by the Centers for Disease Control and Prevention (USA), report that rates of SSI, from individual institutions, range from $0 \%$ to $15 \%$, depending on the indication to surgery, site, approach, and the instrumentation $[4,13]$. Olsen et al. [13] report that the incidence of SSI in their series following orthopedic spinal operations is $2.0 \%$ ( 46 of 2,316 cases presented).

\section{Risk Factors}

There are numerous influences on the development of postoperative infection that could be divided in unchangeble, strictly patient-related, and changeable or procedure related [14-16]. The so-called unchangeable risk factors include patient's age (older than 70 years), ASA score and medical conditions of which the most important are diabetes mellitus, cardiovascular disease, obesity, smoking, malignancy, steroid use, previous lumbar surgery, nutritional status, chronic obstructive pulmonary disease, immunologic competency [13,15-17]. The nutrition status of the patient should be considered as a standalone risk factor [14]. Several authors consider a low blood albumin level and a low white blood cell count as proof of a weakened immune system and therefore a risk factor for infection. Duration of surgery, estimated blood loss, transfusions, use of instrumentation, multiple staged interventions, amount of levels fused, duration of patient stay in the postanesthesia care unit and prolonged preoperative hospital stay are the most important changeable risk factors [15-20].

Koutsoumbelis et al. [15] show that a large number of people in the operating room during the surgical procedure, specifically the number of nurses, is a risk factor for infection. Not all risk factors can be eliminated, therefore a zero rate outcome cannot be expected for the risk of hospital-acquired infections but preoperative modification of the changeable risk factors can lead to alteration of a patient's overall risk and most likely improve his/her outcomes [16,21].

\section{Classification}

From an anatomical point of view, SSI's can be classified as superficial and deep. Superficial infections are limited only to the skin or subcutaneous tissues without fascial involvement $[1,8,16]$. Deep infections involve the fascia and/or muscle. Superficial wound infections present with pain, redness, swelling, warmth, and drainage but are deficient of systemic symptoms and signs $[8,16,22]$. Diskitis, osteomyelitis or epidural abscess are considered consequences of deep infections [8].

Chronologically, SSI's can be classified as early, if they occur within 3 weeks of the procedure, or delayed if they occur more than 4 weeks later $[8,16,22]$. It is also important to distinguish between infections occurring in adult and pediatric patients [7].

Thalgott et al. [3] propose another interesting but more complex classification based on the number of microorganisms isolated, i.e., group 1with presence of a single type, group 2 with presence of multiple types and group 3 with multiple types with myonecrosis; in addition, they are simultaneously divided on the basis of the functional status of the patient into groups A, B, and C, i.e., normal, with local or multiple systemic disease and immunocompromized, respectively. The combination of letters and numbers describes the severity of the infection. The aim of this classification is to facilitate the choice of treatment on the basis of infection severity considered from a strictly microbiological point of view and the host immune response.

\section{Prevention}

Prevention of SS infection in spine surgery includes a set of wide variety of measures directed to the reduction of all the changeable risk factors mentioned earlier. Firstly, it is clear that the choice of intervention based on the risk of postoperative infections is crucial; therefore, when applicable, a less invasive method should be encouraged. The same attitude should be adopted with regard to the use of instrumentation. It is recommended that all distant site infections be aggressively treated in the perioperative period to reduce SSI. The use of perioperative antibiotic prophylaxis to prevent infections is widespread and leads to a reduction of postoperative spinal infections to $<6 \%$ [16].

Generally, a first-generation cephalosporin is used; and 
for patients allergic to this kind of medication, vancomycin, clindamycin, or ciprofloxacin are useful alternatives $[1,2,5,23,24]$. Other studies show that perioperative antibiotics have a 10 fold decrease risk for postoperative infection after discectomy $[1,16,24,25]$. Patients with instrumented fusions have a decreased infection rate with the use of prophylaxis, as compared with patients having surgery without prophylaxis $[1,2,14,23,25]$.

The study by Rubinstein et al. [14], to our knowledge, is the only study that focuses on perioperative antibiotic prophylaxis; they report that the use of perioperative prophylactic dose of cephazolin in spinal surgery do not reduce the incidence of postoperative infections at all but reduce the severity of the infection eventually occurred. Other authors consider that the excessive use of antibiotic prophylaxis in every surgical intervention has the risk of developement of bacterial resistance [14,23].

In the prevention of postoperative infections, sterile technique in operating room is extremely important. For a clinical infection to occur at the surgical site, bacteria must be present at the operative/procedural site in substantial quantity ( $10^{5}$ organisms). It is widely recognized that most postprocedural infections are a consequence of direct inoculation and thus meticulous operative technique is required [8].

The invasiveness and complexity of the procedure is directly related to the infective complications rate. Accordingly, Gelalis et al. [18] took microbial samples for culture from surgical wounds during surgery at regular intervals and report no significant difference in the intraoperative contamination rate of the wound between shorter procedures, usually less invasive, and longer interventions, usually more complex.

Different adjunctive measures reportedly reduce the incidence of postoperative infections, for example, the use of chemical agents, as local antibiotics and disinfectants, or physical agents as prophylactic drains [1,26-28]. The use of powdered vancomycin locally administered during surgery is an effective prophylactic measure to reduce the incidence of postoperative spinal wound infections [29]. The literature shows that adjunctive local application of vancomycin powder decreases the postsurgical wound infection rate with statistical significance in posterior instrumented thoracolumbar spine fusions. Cheng et al. [30] report the efficacy of dilute betadine solution irrigation in the prevention of postoperative infection of spinal surgery. The use of prophylactic drains is com- mon in orthopedics [26-28]. The theoretical advantages of postoperative drain use include the evacuation of the postoperative hematoma and seroma, thereby decreasing the risk of infection and wound breakdown $[26,28]$. In contrast to this commonly accepted principle, Brown and Brookfield [26] report no difference between patients with or without drainage placement. The use of drains is not recommended as a means to reduce infection rates after single-level surgical procedures into the North American Spine Society's evidence-based guidelines for antibiotic prophylaxis in spine surgery [23].

It is a very common practice to prepare the skin before surgery by shaving the skin at the level of incision. Celik and Kara [31] questioned whether skin shaving of the incision site could decrease the risk of infection and they demonstrate that, a cohort of 789 patients who received pre-surgical skin shaving have higher infection rate than patients who do not. This could be explained in part by assessing whether shaving can cause a change in or loss of protective skin flora at the incision line, and microtrauma of the shaved area can increase bacterial colonization; both of these hazards can increase the risk of infection [31].

\section{Microbiology}

The most frequent microorganism found in spinal SSI is Staphylococcus aureus $[1,9,15,24]$. Staphylococcus epidermidis and Enterococcus species have also been noted with increasing frequency in postoperative infections $[1,24]$. Patients with compromised immune system could present with surgical wound infected by low virulence microorganisms [17].

In the last few years, there has been a relative increase in other organisms, particularly gram-negative [24]; several authors report that the routine use of prophylactic antibiotics recently has altered the spectrum of organisms reported to cause postoperative spinal infections $[13,16]$.

\section{Diagnosis of Spinal Infections}

White blood cell count is an unreliable indicator of infection. The acute phase reactants are more useful for diagnosing infection but must be interpreted with respect to the time since the index surgery. Erythrocyte sedimentation rate can remain elevated for up to 6 weeks after surgery. C-reactive protein (CRP) levels normalize within 
2 weeks $[1,8,9,11,16]$. Consequently, CRP is a more sensitive indicator of the presence of SSI. Superficial cultures, whether from the skin or drainage, do not reliably assist with identification of the causative organism. Wound aspiration has been proposed by some authors as a way to detect early infections. However, intraoperative tissue cultures remain the gold standard for identification of the causative organism in SSI $[1,9,11,16]$.

\section{Imaging in Spinal Infections}

Plain radiographs of the spine are rarely useful for the diagnosis of early infection $[8,9,16]$. In the setting of discitis, there may be evidence of loss of disc height and end plate erosion. In latent infections, lucencies may be present around orthopedic hardware. Magnetic resonance imaging (MRI) is the most useful study to diagnose SSI $[1,8-10,16]$. Gadolinium enhancement improves the diagnostic accuracy of MRI and should be used whenever infection is suspected. Findings must be interpreted based on the timing since the index procedure and other potentially confounding conditions since tissue edema from noninfectious causes may mimic the appearance of infection. Rim enhancing fluid collections, ascending epidural collections, evidence of bony destruction, and progressive marrow signal changes are all suggestive of infection (Fig. 1). If infection is suspected in patients with hardware in the spine, computed tomography scan done at an early stage can detect lucency around orthopedic implants (Fig. 2).

\section{Treatment}

SSI in spine surgery can be difficult to manage and often necessitate prolonged hospitalization, extended use of antibiotic therapy, wound debridement and irrigation or instrumentation removal $[4,17]$. The traditional protocol treatment of spinal SSI is early recognition, debridement, irrigation and culture-specific antibiotic administration.

Usually, immediately after the diagnosis of deep wound
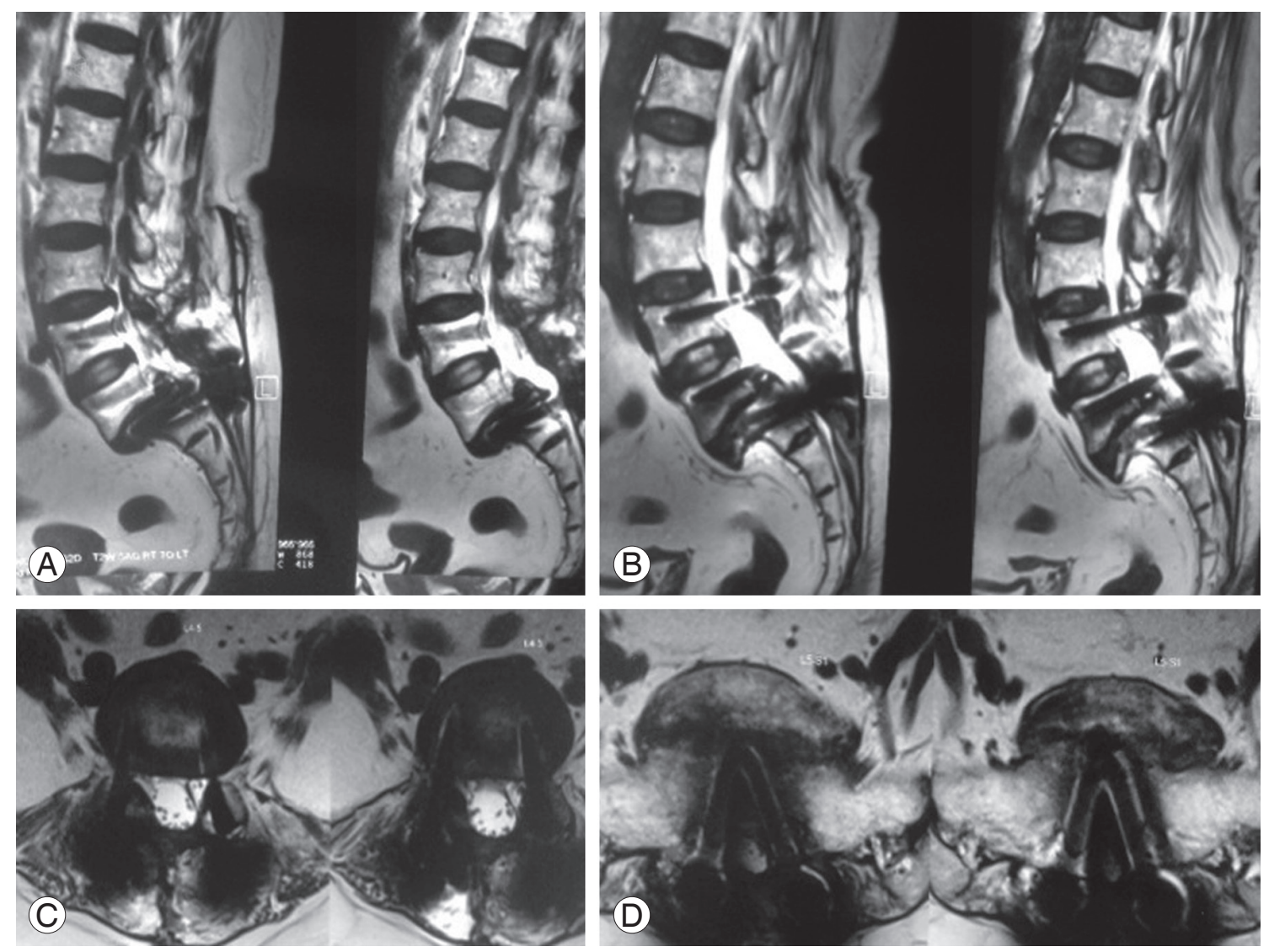

Fig. 1. (A-D) Magnetic resonance imaging sections in patients with postoperative infection after instrumentation showing marrow signal changes and fluid collection. 

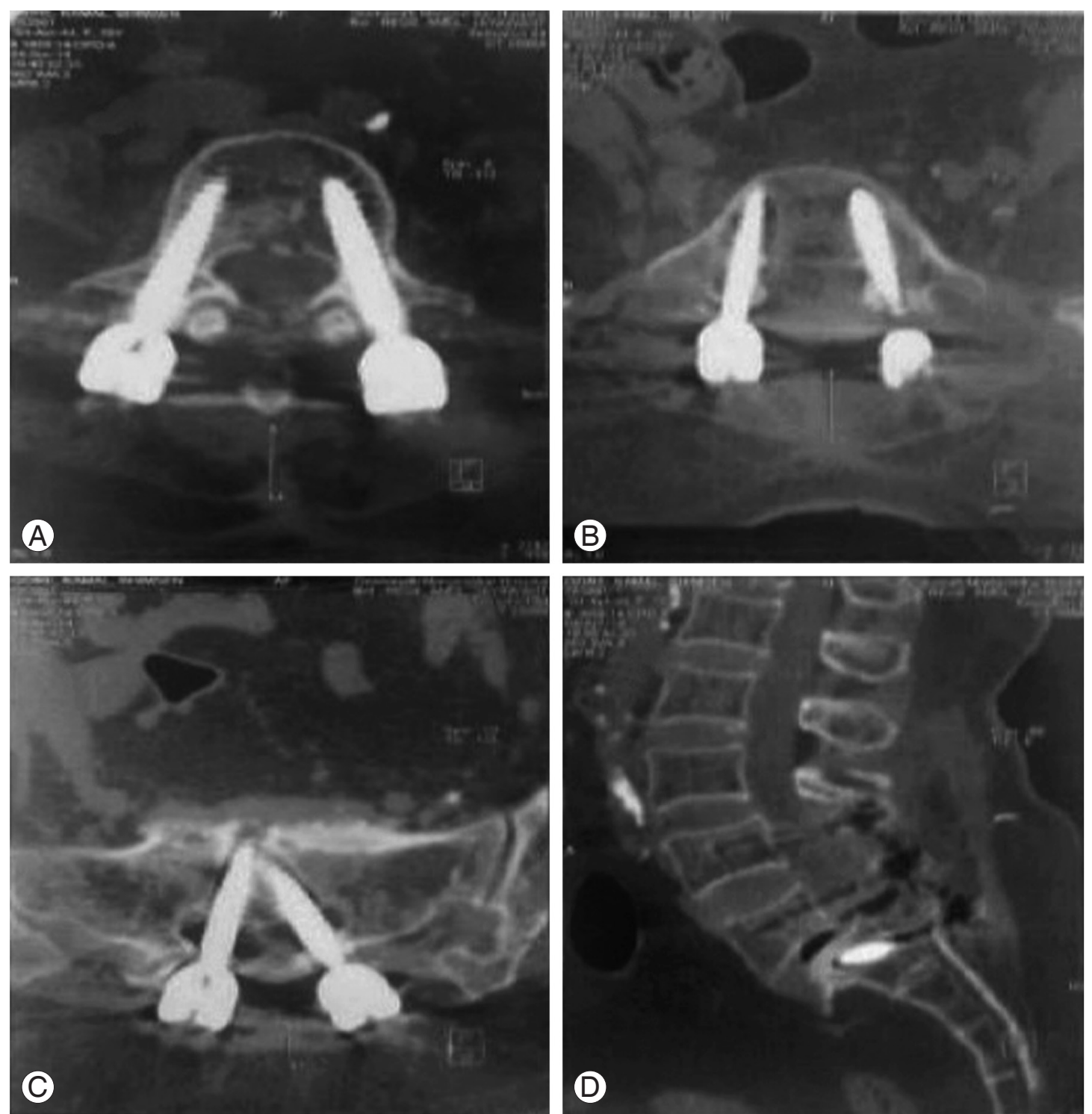

Fig. 2. (A-D) Computed tomography scan in the same patient showing lucency around spinal hardware.

infection, the patient is brought to the OR where the wound is thoroughly debrided and irrigated under general anesthesia; if the tissue has no necrosis and is clean at the end of the debridement, the wound is closed over suction drain $[1,17,32-34]$. If after the debridement the tissue looks questionable, the wound is packed open and redebrided in 2 to 4 days. In case of infection with a delayed onset of more than 37 weeks, the instrumentation can be removed because the arthrodesis should be solid [32-34].

Surgical management of deep wound infections is mandatorily associated with long term antibiotic therapy [1,32-34]. Many authors agree that it is always useful to obtain a microbial culture of the infected wound to start a specific intravenous antibiotic therapy [1]. Weinstein et al. [1] suggest that definitive treatment depends on the culture results but generally, they consider infections with anaerobes as polymicrobial to be treated with i.v., broadspectrum antibiotics, such as vancomycin or metronidazole, administered i.v., for 6 weeks. Suppressive antibiotic therapy usually consists of oral administration of sulfamethoxazole-thrimethroprim or doxycyline; this suppressive antibiotic therapy is not necessary for sensitive S. aureus and E. coli. The suppressive antibiotic therapy is different for anaerobes that are sensitive to orally administered suppressive doses of amoxicillin [33,35].

Recently, the use of vaccum assisted closure (V.A.C.) 
dressings and closed suction irrigation systems is becoming more popular in the clinical management of infected wounds to support the wound healing process $[33,34,36]$. Rohmiller et al. [36] are of the opinion that the advent of negative pressure suction devices has changed the management of postoperative wound infections dramatically. They report a series of 28 postoperative deep wound infections treated with debridment, irrigation and placement of a continous suction and irrigation system with the use of a sterile saline normal solution for a mean period of 4 days. No antibiotic was added to the solution. All infected wounds healed without the need of any additional measure. On the other hand, Labler et al. [33] have contradictory findings. They report that the drainage of an open wound under negative pressure V.A.C. dressing is more efficient than local irrigation systems by continuously removing the wound fluid, which inhibits mitosis, protein synthesis and fibroblast collagen synthesis and prevents its stasis in the wound. The drainage of extracellular fluid also reduces the interstitial pressure, increases blood flow and thus the local nutrition as well; they report good results in a series of 15 postoperative wound infections of the dorsal spine treated with multiple debridement, irrigation, i.v., antibiotic therapy and placement of V.A.C. dressing. In addition, Mehbod et al. [34] proposed the application of V.A.C. devices as a useful method to decrease the number of visits to the OR for debridment. They report a 2.2 average number of visits to the OR after the application of the Vaccum Assisted Closure dressing, as compared to the previously reported average between 2.7 and 4.7 .

The treatment algorithm of Abbey et al. [35] is currently valid in the planning of treatment strategy. They suggest that the instrumentation should be removed only when multiple debridement and long period antibiotic therapy fail to result in the recovery from infection; on the contrary, Picada et al. [32] state that instrumentation removal is not necessary in acute infections and that the instrumentation should remain in place until achieving the desired immobilization for arthrodesis.

\section{Conclusions}

Despite all the measures to reduce the incidence of SSI in spine surgery, these remain a dangerous complication. Prevention is the best way to solve the problem; the risk factors of each patient should be analyzed and the change- able ones should be eliminated.

In both, the short and long postsurgical period, surveillance by the surgeon is vital to an early diagnosis; once the infection is diagnosed, a treatment strategy should be planned. The key points of treatment are aggressive surgical treatment and targeted antibiotic therapy to eradicate the infection and limit damage to local tissues including the neural elements.

\section{Conflict of Interest}

No potential conflict of interest relevant to this article was reported.

\section{References}

1. Weinstein MA, McCabe JP, Cammisa FP Jr. Postoperative spinal wound infection: a review of 2,391 consecutive index procedures. J Spinal Disord 2000;13: 422-6.

2. Veeravagu A, Patil CG, Lad SP, Boakye M. Risk factors for postoperative spinal wound infections after spinal decompression and fusion surgeries. Spine (Phila Pa 1976) 2009;34:1869-72.

3. Thalgott JS, Cotler HB, Sasso RC, LaRocca H, Gardner V. Postoperative infections in spinal implants. Classification and analysis: a multicenter study. Spine (Phila Pa 1976) 1991;16:981-4.

4. Horan TC, Culver DH, Gaynes RP, Jarvis WR, Edwards JR, Reid CR. Nosocomial infections in surgical patients in the United States, January 1986-June 1992. National Nosocomial Infections Surveillance (NNIS) System. Infect Control Hosp Epidemiol 1993;14:7380.

5. Pull ter Gunne AF, Cohen DB. Incidence, prevalence, and analysis of risk factors for surgical site infection following adult spinal surgery. Spine (Phila Pa 1976) 2009;34:1422-8.

6. Fang A, Hu SS, Endres N, Bradford DS. Risk factors for infection after spinal surgery. Spine (Phila $\mathrm{Pa}$ 1976) 2005;30:1460-5.

7. Smith JS, Shaffrey CI, Sansur CA, et al. Rates of infection after spine surgery based on 108,419 procedures: a report from the Scoliosis Research Society Morbidity and Mortality Committee. Spine (Phila Pa 1976) 2011;36:556-63.

8. Chaudhary SB, Vives MJ, Basra SK, Reiter MF. Post- 
operative spinal wound infections and postprocedural diskitis. J Spinal Cord Med 2007;30:441-51.

9. Heller JG. Postoperative infections of the spine. In: Rothman RH, Simeone FA, editors. The spine. Philadelphia: WB Saunders; 1992. p.1817-37.

10. Massie JB, Heller JG, Abitbol JJ, McPherson D, Garfin SR. Postoperative posterior spinal wound infections. Clin Orthop Relat Res 1992;(284):99-108.

11. Bassewitz HL, Fishgrund JS, Herkowitz HN. Postoperative spine infections. Semin Spine Surg 2000;12: 203-11.

12. Dubousset J, Shufflebarger HL, Wenger D. Late "infection" with CD instrumentation. Orthop Trans $1994 ; 18: 121-8$

13. Olsen MA, Nepple JJ, Riew KD, et al. Risk factors for surgical site infection following orthopaedic spinal operations. J Bone Joint Surg Am 2008;90:62-9.

14. Rubinstein E, Findler G, Amit P, Shaked I. Perioperative prophylactic cephazolin in spinal surgery: a double-blind placebo-controlled trial. J Bone Joint Surg Br 1994;76:99-102.

15. Koutsoumbelis S, Hughes AP, Girardi FP, et al. Risk factors for postoperative infection following posterior lumbar instrumented arthrodesis. J Bone Joint Surg Am 2011;93:1627-33.

16. Christodoulou AG, Givissis P, Symeonidis PD, Karataglis D, Pournaras J. Reduction of postoperative spinal infections based on an etiologic protocol. Clin Orthop Relat Res 2006;444:107-13.

17. Cunningham ME, Girardi F, Papadopoulos EC, Cammisa FP. Spinal infections in patients with compromised immune systems. Clin Orthop Relat Res 2006; 444:73-82.

18. Gelalis ID, Arnaoutoglou CM, Politis AN, Batzaleksis NA, Katonis PG, Xenakis TA. Bacterial wound contamination during simple and complex spinal procedures: a prospective clinical study. Spine J 2011; 11:1042-8.

19. Klein JD, Hey LA, Yu CS, et al. Perioperative nutrition and postoperative complications in patients undergoing spinal surgery. Spine (Phila Pa 1976) 1996; 21:2676-82.

20. Dick J, Boachie-Adjei o, Wilson M. One-stage versus two-stage anterior and posterior spinal reconstruction in adults comparison of outcomes including nutritional status, complication rates, hospital costs, and other factors. Spine (Phila Pa 1976);17(8 Suppl):S310-6.
21. Schuster JM, Rechtine G, Norvell DC, Dettori JR. The influence of perioperative risk factors and therapeutic interventions on infection rates after spine surgery: a systematic review. Spine (Phila Pa 1976) 2010;35:S125-37.

22. Sasso RC, Garrido BJ. Postoperative spinal wound infections. J Am Acad Orthop Surg 2008;16:330-7.

23. Watters WC 3rd, Baisden J, Bono CM, et al. Antibiotic prophylaxis in spine surgery: an evidence-based clinical guideline for the use of prophylactic antibiotics in spine surgery. Spine J 2009;9:142-6.

24. Horwitz NH, Curtin JA. Prophylactic antibiotics and wound infections following laminectomy for lumber disc herniation. J Neurosurg 1975;43:727-31.

25. Barker FG 2nd. Efficacy of prophylactic antibiotic therapy in spinal surgery: a meta-analysis. Neurosurgery 2002;51:391-400.

26. Brown MD, Brookfield KF. A randomized study of closed wound suction drainage for extensive lumbar spine surgery. Spine (Phila Pa 1976) 2004;29:1066-8.

27. Payne DH, Fischgrund JS, Herkowitz HN, Barry RL, Kurz LT, Montgomery DM. Efficacy of closed wound suction drainage after single-level lumbar laminectomy. J Spinal Disord 1996;9:401-3.

28. Beer KJ, Lombardi AV Jr, Mallory TH, Vaughn BK. The efficacy of suction drains after routine total joint arthroplasty. J Bone Joint Surg Am 1991;73:584-7.

29. Molinari RW, Khera OA, Molinari WJ 3rd. Prophylactic intraoperative powdered vancomycin and postoperative deep spinal wound infection: 1,512 consecutive surgical cases over a 6-year period. Eur Spine J 2012;21 Suppl 4:S476-82.

30. Cheng MT, Chang MC, Wang ST, Yu WK, Liu CL, Chen TH. Efficacy of dilute betadine solution irrigation in the prevention of postoperative infection of spinal surgery. Spine (Phila Pa 1976) 2005;30:168993.

31. Celik SE, Kara A. Does shaving the incision site increase the infection rate after spinal surgery? Spine (Phila Pa 1976) 2007;32:1575-7.

32. Picada R, Winter RB, Lonstein JE, et al. Postoperative deep wound infection in adults after posterior lumbosacral spine fusion with instrumentation: incidence and management. J Spinal Disord 2000;13:425.

33. Labler L, Keel M, Trentz O, Heinzelmann M. Wound conditioning by vacuum assisted closure (V.A.C.) in 
postoperative infections after dorsal spine surgery. Eur Spine J 2006;15:1388-96.

34. Mehbod AA, Ogilvie JW, Pinto MR, et al. Postoperative deep wound infections in adults after spinal fusion: management with vacuum-assisted wound closure. J Spinal Disord Tech 2005;18:14-7.

35. Abbey DM, Turner DM, Warson JS, Wirt TC, Scalley RD. Treatment of postoperative wound infections following spinal fusion with instrumentation. J Spinal Disord 1995;8:278-83.

36. Rohmiller MT, Akbarnia BA, Raiszadeh K, Raiszadeh $\mathrm{K}$, Canale S. Closed suction irrigation for the treatment of postoperative wound infections following posterior spinal fusion and instrumentation. Spine (Phila Pa 1976) 2010;35:642-6. 